\title{
The financial impact of an Enhanced Recovery Protocol in colo-rectal surgical care
}

\author{
Nathan M. Johnson*1, Sandy L. Fogel ${ }^{2}$ \\ ${ }^{1}$ Virginia Tech Carilion School of Medicine, Roanoke, VA, United States \\ ${ }^{2}$ Department of Surgery, Carilion Clinic, Roanoke, VA, United States
}

Received: October 4, 2015

Accepted: December 8, 2015

Online Published: December 21, 2015

DOI: $10.5430 /$ jha.v5n2p23

URL: http://dx.doi.org/10.5430/jha.v5n2p23

\begin{abstract}
Objective: Enhanced Recovery Protocols (ERPs) have been shown in many different settings to lead to quicker recovery for most patients, with a significantly reduced average length of post-operative stay (LOS). A less studied impact of ERPs has been their effect on hospital profitability. While these protocols are resource-intensive and expensive to implement, we argue that they can lead to significantly improved margins. This can be attributed to fewer complications and, more significantly, reductions in LOS resulting in increased patient capacity.

Methods: Our ERP was implemented in June of 2014. The protocol was initially used only for colo-rectal cases, both elective and emergent. It contained over 20 pre-, intra-, and post-operative elements of surgical care. One year of data from the ERP cases was compared to contemporaneous controls that did not go through the ERP. Financial data was obtained from the hospital cost accountant. Average LOS was obtained from the EHR.

Results: Patients who underwent colo-rectal procedures and participated in the ERP had an average LOS of 5.60 days, while controls stayed for an average of 8.51 days. Financial analysis determined that a full year of compliance with Enhanced Recovery After Surgery (ERAS) protocols added over 2 million dollars to the margin for a return on investment (ROI) of over 10 to 1 , mainly by increasing hospital capacity and allowing more admissions.

Conclusions: The results demonstrate that ERPs significantly reduce LOS, increasing hospital patient capacity. The higher patient load more than recoups ERP costs. Further collection and analysis of data aims to determine the effect on complications, which also have cost saving potential.
\end{abstract}

Key Words: Enhanced Recovery Protocol, Enhanced Recovery After Surgery, Fast tracking, Quality improvement, Costeffectiveness

\section{INTRODUCTION}

Enhanced Recovery Protocols (ERPs), also known as Enhanced Recovery After Surgery (ERAS), are evidence-based approaches to peri-operative surgical care aimed at attenuating the stress response following surgery, promoting early return of function, and minimizing common post-operative complications. ERPs were pioneered in the setting of colo- rectal procedures ${ }^{[1]}$ but have since been studied in urological, ${ }^{[2]}$ thoracic, ${ }^{[3]}$ and vascular procedures ${ }^{[4]}$ among others. While no two ERPs are identical, most contain preoperative components that serve to optimize both physiologic and psychological function prior to surgery, intra- and post-operative techniques that promote maintenance of organ function and minimization of the surgical stress response,

\footnotetext{
*Correspondence: Nathan M. Johnson; Email: nmjohnson@ carilionclinic.org; Address: Nathan Johnson, Virginia Tech Carilion School of Medicine, Roanoke, VA 24016, United States.
} 
and peri-operative measures that reduce the risk of common complications. Common themes include preadmission counseling and nutritional optimization, utilization of thoracic epidurals, minimal use of narcotics for anesthesia/analgesia, avoidance of salt and water overload, and early stimulation of organ function.

Numerous studies have demonstrated the effectiveness of ERPs in reducing post-operative length of hospital stay (LOS) in colo-rectal procedures, which serves as a surrogate for common discharge criteria, such as return of bowel function, tolerance of diet, return of activity, and control of post-operative pain. ${ }^{[1,5,6]}$ A 2011 systematic review of ERPs in colo-rectal surgery noted an average LOS reduction of 2.53 days for ERP patients as compared to those managed with traditional care pathways. Additionally, an increasing number of studies are exploring the role of ERPs in the prevention of common post-operative complications, with promising results. ${ }^{[7]}$

While the ERP movement is slowly gaining steam, implementation of ERPs remains fairly low despite these welldocumented improvements in outcomes. This may be due to the challenge they pose to entrenched surgical practices and the necessity of considerable resource inputs - both personnel and monetary. Indeed, questions raised by critics often focus on the sustainability of ERPs from a cost perspective. Although there are still relatively few studies on this issue, a 2010 study by Sammour et al. demonstrated that implementing an ERAS program offset its input costs by reducing resource utilization in the post-operative period alone, ${ }^{[8]}$ and a more recent 2013 article by Roulin et al. further examined cost-effectiveness of ERPs in colo-rectal surgery, finding their program to be cost-effective, even in the initial imple- mentation period. ${ }^{[9]}$ And a further study by Michard et al. (in press) comes to the same conclusion using a very different methodology, including a very large data base and expected savings from reductions in complications after employing peri-operative goal-based fluid therapy, one of the cornerstones of ERPs. ${ }^{[10]}$ Our study builds on this growing body of evidence that well-developed and executed ERPs are costeffective. It also explores the potential for ERPs to increase revenue generation for the institution, further building the case for adoption of ERPs into surgical practice.

\section{Methods}

Carilion Roanoke Memorial Hospital (CRMH) is a busy, 783-bed tertiary care facility in southwestern Virginia. The hospital is frequently on diversion for lack of capacity. In fiscal year 2014, 817 transfers were declined as a result, with an ensuing loss of the income that those patients would have generated.

Carilion's ERP was implemented in colo-rectal procedures in June of 2014. This study reflects one year of ERP implementation data from July 1, 2014 through June 30, 2015. During the study period, the subgroup of patients who did not participate in the protocol served as contemporaneous controls. The patient populations are roughly comparable (see Table 1), with none of the differences reaching statistical significance at the .05 level. LOS served as the main variable for this study as it has the greatest impact on cost and costrecovery for surgical procedures. Other variables, including complications, readmissions, returns to the operating room, and total morbidity and mortality were also collected, but proper and meaningful analysis of these variables requires more than one year of data.

Table 1. Comparison between the enhanced recovery patients and the non-enhanced recovery patients

\begin{tabular}{llllllll}
\hline Risk Factor & Avg age & Avg BMI & Avg ASA & Hx MI (\%) & Hx CHF (\%) & Dyspnea (\%) & Diabetes (\%) \\
\hline ERP patients & 56.1 & 29.1 & 2.55 & $6.0 \%$ & $1 \%$ & $9.2 \%$ & $17.9 \%$ \\
Non-ERP patients & 61.1 & 27.6 & 2.57 & $10.7 \%$ & $1.5 \%$ & $10.7 \%$ & $23.1 \%$ \\
\hline
\end{tabular}

Note. No difference reached statistical significance at the .05 level; ERP: Enhanced Recovery Protocols; BMI: body mass index; ASA: American Society of Anesthesiologists; Hx MI: history of myocardial infarction; Hx CHF: history of congestive heart failure

The specific ERP designed and implemented at Carilion included 28 official "elements" of care. A complete listing can be seen in Figure 1. The five major components of the protocol include pre-operative care, multimodal anesthesia/analgesia, peri-operative goal-directed fluid management, early post-operative stimulation of function, and complication prophylaxis. The pre-operative component involves an educational class that serves to reduce patient anxiety and optimize function leading up to the procedure. Patients are given resources and instructed to focus on proper nutrition, exercise, oral hygiene, smoking cessation, incentive spirometry, and the use of statins in appropriately selected patients. ${ }^{[11,12]}$ Standardized, multimodal analgesia/anesthesia - utilizing epidurals, nerve blocks, and non-narcotic analgesics - is another key component of the protocol, since successful pain control is essential to attenuating the surgical stress response, and narcotic use can delay recovery and return of bowel function. ${ }^{[13]}$ The protocol also employs peri- 
operative goal-directed fluid therapy, which aims to eliminate the great variability in fluid management during colo-rectal procedures. This is achieved via the use of minimally in- vasive cardiac output monitoring in order to minimize both hypovolemia and fluid overload, both of which are associated with increased rates of post-operative complications. ${ }^{[14]}$

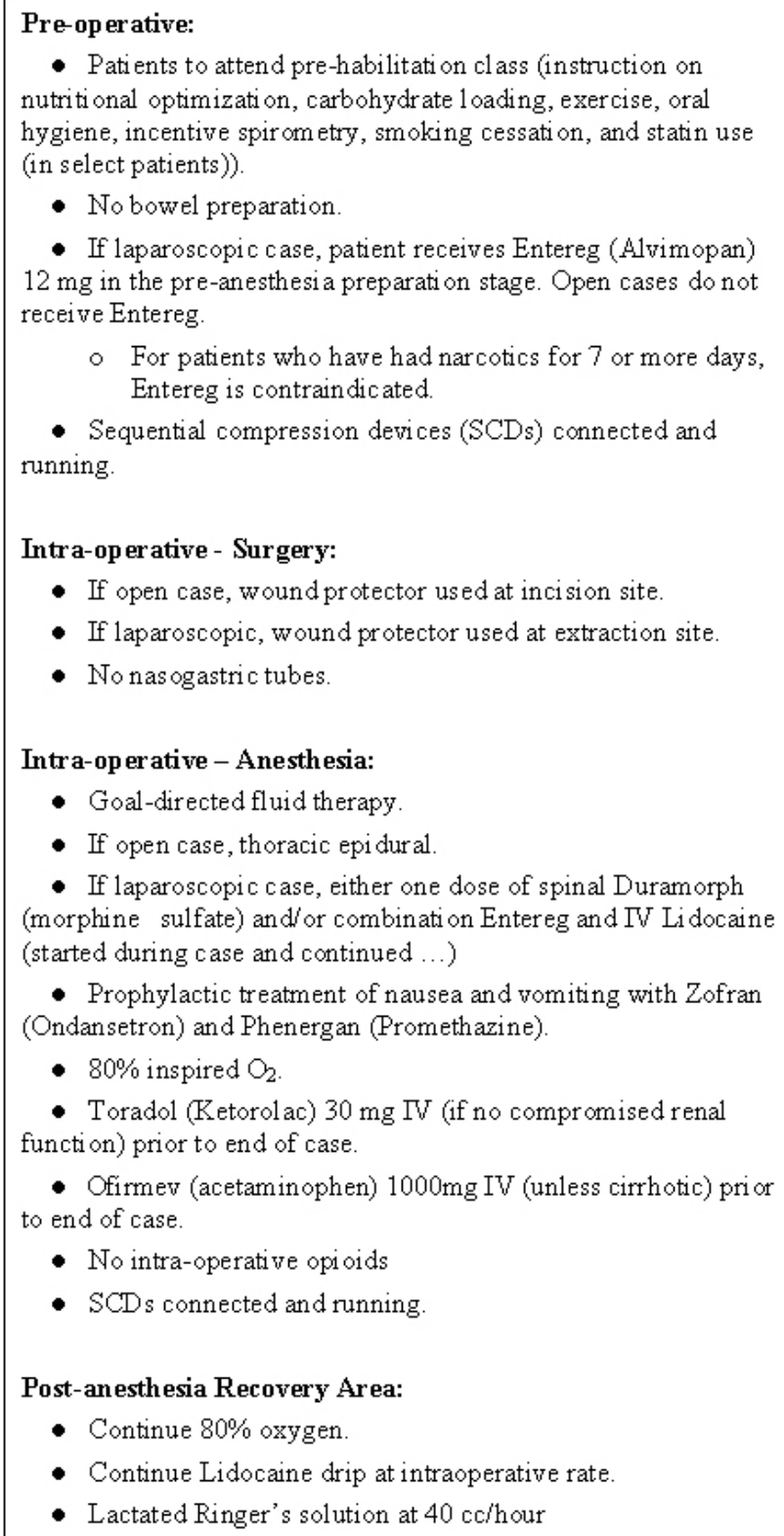

- Patients to attend pre-habilitation class (instruction on nutritional optimization, carbohydrate loading, exercise, oral hygiene, incentive spirometry, smoking cessation, and statin use (in select patients))

- Nobowel preparation

- If laparoscopic case, patient receives Entereg (Alvimopan) $12 \mathrm{mg}$ in the pre-anesthesia preparation stage. Open cases do not receive Entereg

- For patients who have had narcotics for 7 or more days, Entereg is contraindicated.

- Sequential compression devices (SCDs) connected and running

Intra-op erative - Surgery:

- If open case, wound protector used at incision site.

- If laparoscopic, wound protector used at extraction site.

- No nas ogastric tubes.

Intra-operative - Anesthesia:

- Goal-directed fluid therapy.

- If open case, thoracic epidural

- If laparoscopic case, either one dose of spinal Duramorph (morphine sulfate) and/or combination Entereg and $\Gamma \mathrm{V} \mathrm{Lidocaine}$ (started during case and continued ...)

- Prophylactic treatment of nausea and vomiting with Zofran (Ondansetron) and Phenergan (Promethazine).

- $80 \%$ inspired $\mathrm{O}_{2}$

- Toradol (Ketorol ac) $30 \mathrm{mg}$ TV (if no compromised renal function) prior to end of case.

- Ofirmev (acetaminophen) 1000mg IV (unless cirrhotic) prior to end of case

- No intra-operative opioids

- SCD s connected and running

\section{Post-anesthesia Recovery Area:}

- Continue $80 \%$ oxygen

- Continue Lidocaine drip at intraoperative rate.

- Lactated Ringer's solution at $40 \mathrm{cc} /$ hour

\section{Post-operative:}

- Epidural management protocol:

- To be removed 8:00 am second moming after surgery.

- Heparin 5000 units subcutaneous BID to start night of surgery for two doses.

- Skip one dose the night before removal.

- Heparin restarted 2 hours after removal, same dose as above.

- If no epidural, use Lovenox (Enoxaparin)

- Entereg in those patients who received it pre-operatively - $12 \mathrm{mg}$ PO BD until first bowel movement or discharge, but no more than 15 total doses.

- Toradol - $30 \mathrm{mg}$ TV every 6 hours for 48 hours - If patient is older than 65 or has compromised renal function, reduced to $15 \mathrm{mg}$.

- Ofirmev - $1000 \mathrm{mg}$ IV q6h for 24 hours, foll owed by oral Tylenol $650 \mathrm{PO}$ q $6 \mathrm{~h}$ for 24 hours (unless cirrhotic).

- Continue Lidocaine drip at same rate for 24 hours

- Early post-operative clear, non-carbonated liquids by mouth (day of surgery)

- Limit post-operative IV fluids to $40-60 \mathrm{cc} / \mathrm{hr}$.

- Limit fluid boluses to only those with symptomatic hypotension or tachycardia.

- Add Impact Supplements once tolerating PO well

- Ambulation on the day of surgery and at least 4 times/day in the post-operative period

- Foley catheter to be removed on the first day unless there is a documented indication for it to remain.

- If patient fails to void, intermittent catheter is used for 24 hours before using another Foley catheter.

- No nasogastric tube.

- SCD s connected and running at all times except when patient is ambulating.

- Incentive spirometry once every hour

Figure 1. Components of carilion clinic's ERP

Our ERP also included prophylactic treatment of nausea with two drugs, early feeding by mouth, and same-day mobilization. Several best practices were added to the protocol to address common complications. These include no use of bowel preparation or nasogastric tubes, use of wound protectors, and early removal of Foley catheters unless there was a documented indication for it to remain.

In terms of data collection, LOS data was obtained directly from the electronic health record (EHR). It should be noted that patients with a LOS greater than 30 days were excluded because of the disproportionate effect they have on averages. During the study period, there were three such patients excluded with LOS up to 151 days. Two were in the ERP group while one not, making the effect of their exclusion negligible in the calculations. Financial data was obtained from the hospital cost accountant. 


\section{RESUltS}

After one year of ERP implementation, a total of 193 colorectal patients had participated in the protocol while $194 \mathrm{had}$ not. This was not randomized, but based upon the preference of the surgeon. However, the groups were roughly comparable as mentioned above. The average LOS for patients participating was 5.60 days, while that of non-participants was 8.51 days. This yields a difference of 2.91 days between the two groups, which is consistent with figures generated in most ERP studies. ${ }^{[8]}$ Statistical significance of these results was established using a simple $T$-test for means, yielding a $p$-value of less than .001.

\section{Results of financial analysis}

The pre-habilitation classes cost $\$ 40$ per patient, while all other costs combined amounted to $\$ 460$ per patient. Multiplying by the possible 400 colo-rectal patients per year yielded the maximum calculated input investment of $\$ 200,000$. The contemporaneous comparison between patients who participated in the ERP and those that did not yielded a difference in LOS of 2.91 days for all cases. Since most hospital payments are under the diagnosis-related groups (DRG) system, the payments per admission are generally fixed. Therefore, shorter LOS generates some savings, but this is small compared to the revenue generated by admissions of new patients.

We used a LOS reduction figure of three days to calculate cost savings and increased revenue generation. The analysis makes several assumptions including average LOS for all admissions to CRMH of 5.07 days, average added revenue of $\$ 6,688$ per new admission, and savings from variable costs of $\$ 343$ per day.

By converting the reduced average LOS information to hospital-bed-days saved and using the internal figure of $\$ 343$ for end-of-hospital-stay cost per day, we calculated that the ERP potentially saved approximately $\$ 400,000$.

More significantly, financial analysis demonstrated that the reduced LOS achieved by implementation of the ERP might allow our institution to accept 240 more patients per year than it otherwise could have because of increased patient capacity. Using the average margin per admission, the analysis determined that this intervention resulted in over 2 million dollars in additional revenue. This reflects a return on investment (ROI) of over 10 to 1 .

If we assume a more conservative 2-day reduction in LOS, it would still allow the system to accept an additional 164 patients per year. This would result in a savings from the reduced LOS of $\$ 285,376$ for the institution and the potential to generate an additional $\$ 1,097,547$ by accepting patients who it would otherwise be unable to accept due to lack of capacity. Totaling the figures and subtracting the input costs $(\$ 200,000)$ yields a 7 to 1 ROI.

\section{Discussion}

Our results demonstrate that ERPs can potentially lead to both cost recovery and substantial revenue generation. Reduced LOS alone represents an area for cost savings, albeit a relatively small one, given that the daily variable cost of an uncomplicated post-operative hospital stay at our institution is approximately $\$ 343$. The true value from a financial perspective of reduced LOS is the resulting increase in patient capacity. This would be so for any hospital that is sufficiently busy to turn down transfers or that might need to delay elective admissions for procedures because of lack of capacity. The results documented should serve as evidence that ERPs are well worth the input costs, as they produce a significant ROI.

ERPs have the potential to further benefit the hospital bottom line in two other areas: incidence of complications and rate of readmissions. According to a 2011 study, thirty-day readmissions occur in approximately $11.4 \%$ of patients undergoing colo-rectal surgery, and an additional $11.9 \%$ are readmitted within 90 days of their procedure. On average, these patients were hospitalized for 8 days per readmission, costing the health system $\$ 8,885$ each. ${ }^{[15]}$ Clearly, readmissions represent an opportunity for quality improvement and cost savings, as readmissions for complications or failed procedures decrease bed space and potentially reduce Medicare reimbursements. Historically, there has been concern that ERP implementation might increase readmissions, but the literature, including a 2011 systematic review, has demonstrated that this has not been the case. ${ }^{[7]}$ More recent studies not only reinforce this conclusion, but also suggest that ERPs have the potential to reduce the rate of readmissions in colorectal procedures. ${ }^{[16]}$

The cost burden of post-operative complications is highly variable and challenging to accurately quantify, but it is arguably an area ripe for quality improvement and further cost savings. While the role of ERPs in reducing complications remains an area that requires more investigation, several studies have demonstrated reductions in certain complication rates, ${ }^{[17]}$ which is unsurprising given that many ERPs, including this one, contain elements specifically addressing this goal. While more research is required, both of these areas represent potential benefits of ERPs in terms of cost-savings which serve to further support the case for implementation of ERPs.

It is also important to acknowledge the limitations involved in the implementation of Carilion Clinic's ERP. Given the 
authors' assertion that ERPs lead to improved outcomes, randomization to treatment and control groups was not possible, and the study, therefore, relied on physicians opting out of the protocol to establish a contemporary control group. This introduces a difficult to quantify bias in the selection of the intervention and control groups. Although the two groups are roughly similar, subtle selection bias cannot be totally excluded. Additionally, the protocol was quite complex and adherence to every component as well as proper documentation was difficult. Initially, many surgeons and anesthesiologists were quite reluctant to participate, which accounts for a rate of compliance with all components of the protocol of approximately $50 \%$. Of note, compliance with the protocol improved dramatically over the course of the study period as physicians and nursing staff became more familiar with the protocol, a process that was aided by specific educational interventions throughout the year. It is reasonable to assume that improving adherence to all components of the protocol might result in an even greater effect on LOS, and thus greater cost-saving potential. Finally, this study rests on the assumption that demand exceeds available resources; more specifically, that patients are being turned away due to lack of availability, so greater availability would lead to more revenue generation. While this is the case at our institution, a busy tertiary care center, it should be acknowledged that it may not be the case at some institutions.

It should be remembered that, by far, the strongest evidence and motive for implementing ERPs in colo-rectal procedures is their effect on patient care. In general, patients participating in ERPs recover more quickly and are able to return to daily activities sooner. Further, these patients experience less anxiety and fewer complications prior to and after their procedures. The additional benefit that ERPs potentially have for hospital cost-savings is merely a bonus - one that hopefully serves to convince even more physicians, patients, hospital executives, quality improvement champions, and other stakeholders to seriously consider exploring ERPs and their ability to improve peri-operative surgical care.

\section{REFERENCES}

[1] Wind J, Polle SW, Fung Kon Jin PH, et al. Systematic review of enhanced recovery programmes in colonic surgery. Br J Surg. 2006 Jul; 93(7): 800-9. PMid: 16775831. http://dx. doi .org/10.10 $02 /$ bjs. 5384

[2] Arumainayagam N, McGrath J, Jefferson KP, et al. Introduction of an enhanced recovery protocol for radical cystectomy. BJU Int. 2008 Mar; 101(6): 689-701. PMid: 18190646. http://dx.doi.org/10. 1111/j.1464-410X.2007.07319.x

[3] Tovar EA, Roethe RA, Weissig MD, et al. One-day admission for lung lobectomy: an incidental result of a clinical pathway. Ann Thorac Surg. 1998 Mar; 65(3): 803-6. http://dx.doi.org/10.1016 /S0003-4975(97)01381-7

[4] Podore PC, Throop EB. Infrarenal aortic surgery with a 3-day hospital stay: A report on success with a clinical pathway. J Vasc Surg. 1999 May; 29(5): 787-92. http://dx.doi .org/10.1016/S0741 $-5214(99) 70204-1$

[5] Zhuang CL, Ye XZ, Zhang XC, et al. Enhanced recovery after surgery programs versus traditional care for colorectal surgery: a meta-analysis of randomized controlled trials. Dis Colon Rectum. 2013; 56(5): 667-78. PMid: 23575408. http://dx.doi.org/10. 1097/DCR.0b013e3182812842

[6] Miller TE, Thacker JK, White WD, et al. Reduced length of hospital stay in colorectal surgery after implementation of an enhanced recovery protocol. AnesthAnalg. 2014; 118(5): 1052-61. PMid: 24781574. http://dx.doi.org/10.1213/ANE.0000000000000206

[7] Rawlinson A, Kang P, Evans J, et al. A systematic review of enhanced recovery protocols in colorectal surgery. Ann R CollSurg Engl. 2011 Nov; 93(8): 583-8. PMid: 22041232. http://dx.doi .org/10.13 08/147870811X605219

[8] Sammour T, Zargar-Shoshtari K, Bhat Abhijith, et al. A programme of enhanced recovery after surgery (ERAS) is a cost-effective inter- vention in colonic surgery. N Z Med J. 2010 Jul; 123(1319): 61-70. PMid: 20717178.

[9] Roulin D, Donadini A, Gander S, et al. Cost-effectiveness of the implementation of an enhanced recovery protocol for colorectal surgery. BJS. 2013 Jun; 100(8): 1108-14. PMid: 23754650. http: //dx.doi.org/10.1002/bjs. 9184

[10] Michard F, Mountford WK, Krukas MR, et al. Potential return on investment for implementation of perioperative goal-directed fluid therapy in major surgery: a nationwide database study. Perioperative Med (Lond). 2015 Oct; 4: 11. PMid: 26500766. http: //dx.doi.org/10.1186/s13741-015-0021-0

[11] Carli F, Charlebois P, Stein B, et al. Randomized clinical trial of prehabilitation in colorectal surgery. Br J Surg. 2010; 97(8): 1187-1197. PMid: 20602503. http://dx.doi.org/10.1002/bjs.7102

[12] Lindenauer PK, Pekow P, Wang K, et al. Lipid-lowering therapy and in-hospital mortality following major non-cardiac surgery. JAMA. 2004; 291: 2092-2099. PMid: 15126437. http://dx.doi.org/1 $0.1001 /$ jama.291.17.2092

[13] Kehlet H. Effect of postoperative pain on surgical stress response. In Stanley TH, Ashburn A (Eds.), Anesthesiology and pain management: developments in critical care medicine and anesthesiology. Springer Science + Business Media. 1994; 29: 99-103. http://dx.doi.org/10.1007/978-94-011-0816-4_6

[14] Pearse RM, Harrison Da, McDonald N, et al. Effect of a perioperative, cardiac output-guided, hemodynamic therapy algorithm on outcomes following major gastrointestinal surgery: A randomized clinical trial and updated systematic review. JAMA. 2014 Jun; 311(21): 2181-90. PMid: 24842135. http://dx.doi.org/10.1001/jama. 2014.5 305

[15] Wick EC, Shore AD, Hirose K, et al. Readmission rates and cost following colorectal surgery. Dis Colon Rectum. 2011 Dec; 54(12): 1475-9. PMid: 22067174. http://dx.doi.org/10.1097/DCR.0 b013e31822ff8fo 
[16] Lawrence JK, Keller DS, Samia H, et al. Discharge within 24 to 72 hours of colorectal surgery is associated with low readmission rates when using Enhanced Recovery Pathways. J Am Coll Surg. 2013 Mar; 216(3): 390-4. PMid: 23352608. http://dx.doi.org/10. 1016/j.jamcollsurg.2012.12.014
[17] Gustafsson UO, Hausel J, Thorell A, et al. Adherence to the enhanced recovery after surgery protocol and outcomes after colorectal cancer surgery. Arch Surg. 2011; 146(5): 571-7. PMid: 21242424. http://dx.doi.org/10.1001/archsurg.2010.309 